(Rimini)

\title{
HIERONYMI EPISTULA XLVI: \\ PAULAE ET EUSTOCHIAE AD MARCELLAM - DE LOCIS SANCTIS \\ Commentario
}

Dedico questa ricerca a S. E. Mons. Pietro Sambi,

Nunzio Apostolico a Gerusalemme

\section{INTRODUZIONE}

La lettera 46 che porta l'intestazione Paulae et Eustochiae ad Marcellam ${ }^{1}$, tramandata nel corpus delle lettere di Girolamo fino dal secolo VI, è stata variamente datata fino a quando gli studi di cronologia geronimiana di Nautin non hanno stabilito con certezza il 386 come anno di composizione, rivalutando un'ipotesi avanzata quasi tre secoli fa dall'erudito Domenico Vallarsi ${ }^{2}$. Resta difficile tuttavia precisare il mese. Nautin pensa che la lettera sia stata scritta all'inizio della primavera, dopo il ritorno della comitiva di Paola dall'Egitto; qui però non compare alcun accenno a tale viaggio; inoltre, quando

${ }^{1}$ L'epistolario di Girolamo è citato secondo il testo critico predisposto da I. Hilberg per l'edizione CSEL 54-56, Vindobonae - Lipsiae 1910, 1912, 1918; per la lettera 46 tuttavia, ho tenuto presente anche sia l'edizione a cura di D. Vallarsi, Sancti Eusebii Hieronymi Stridonensis Presbyteri Opera, I, Veronae 1734, 198-208, PL 22, 483-493, sia il testo di J.W. Smit in Vita di Martino. Vita di llarione. In memoria di Paola, Milano 1975, $145-237$ (testo), 319-366 (commento); le opere di Girolamo sono citate secondo le più recenti edizioni; mentre sempre a Vallarsi ho fatto ricorso per le opere prive di un testo critico moderno. Per un profilo biografico delle tre pie donne con bibliografia cfr. A. Lippold, Paulys Realencyclopädie der Classischen Alterfumwissenschaft [d'ora in poi PLRE], Suppl. X 508-509, s.v. Paula 2; F. Caraffa, Bibliotheca Sanctorum [d'ora in poi BS] V 302-304, s.v. Eustochio; W. Ensslin, PLRE XIV, 2, 1437, s.v. Marcella 5; G.D. Gordini, BS VIII 644-645, s.v. Marcella.

${ }^{2}$ Per le varianti cfr. CSEL 54, 329; per il copista vedi la precisazione di J. Labourt, Saint Jérôme. Lettres, II, Paris 1951, 100, n. 2; per la datazione della lettera 46 cfr. Sancti Eusebii Hieronymi Stridonensis Presbyteri Opera, I, Veronae 1734, p. [5] e H. Lietzmann, PLRE VIII 2, 1567, s.v. Hieronymus 16 (anno 386); H. Grützmacher, Hieronymus. Eine biographische Studie zur alten Kirchengeschichte, I, Darmstadt 1969, 64-65 (anno 389); F. Cavallera, Saint Jérôme. Sa vie et son oeuvre, II, Louvain -- Paris 1922, p. 43 (anni 392-393); P. Nautin, La lettre de Paule et Eustochium à Marcelle "Augustinianum” 24 (1984) 441-449, seguito da S. Rebenich, Hieronymus und sein Kreis, Stuttgart 1992, 195, n. 324 (primavera del 386). 
Paola tornò in Palestina, dovette imbarcarsi a Pelusio, perché faceva troppo caldo per andare a piedi ${ }^{3}$; se la lettera fosse stata scritta dopo il ritorno dalla terra „fertile di monaci" 4 , non sarebbe stato l'inizio, bensì la fine della primavera o l'inizio dell'estate. Credo perciò che la proposta di Nautin, pur essendo valida, vada suffragata da un'ulteriore considerazione, che la lettera fu scritta all'inizio della primavera, quando Paola e la figlia Giulia (dopo la conversione monastica chiamata Eustochio) insieme con Girolamo dovevano ancora partire per l'Egitto.

La comitiva dei tre illustri personaggi era giunta da Roma a Gerusalemme poco prima della fine del $385^{5}$ in picno inverno, con un grandissimo freddo; insieme avevano compiuto un pellegrinaggio in Palestina, assistendo ai riti pasquali, e avevano scritto velocemente la lettera 46; poi si erano messi di nuovo in cammino alla volta dell'Egitto, dove oltre all'interesse comune per la vita degli anacoreti del deserto, si aggiungeva il vivo desiderio di Girolamo di ascoltare le lezioni di Didimo il Cicco ad Alessandria, presso il quale trascorse poco meno di un mese'.

Per quanto riguarda l'autenticità della lettera, non ci sono elementi di confronto in base ai quali verificare lo stile epistolare di Paola e di Eustochio e quindi potere attribuire con sicurezza la lettcra in questione a una delle due donne; tutta la loro corrispondenza epistolare, come quella di Marcella e delle altre ascete romane, è andata irrimediabilmente perduta ${ }^{7}$. I critici hanno attribuito la lettera a Girolamo sulla base di due soli dati certi: la nota del copista del secolo VI e il riscontro stilistico con le opere di Girolamo ${ }^{8}$, anche se ad entrambi

${ }^{3}$ Cfr. Epistula 108, 14. CSEL 55, 325: „Propter ferventissimos aestus de Pelusio Maiumam navigatione perveniens [sc. Paula]".

${ }_{4}^{4}$ Crr. Episfula 46, 10, CSEL 54, 340 (è l'unico accenno all'Egitto).

5 Cfr. Epistula 108, 9, CSEL 55, 314; P. Nautin, Eudes de chronologie hiéronymienne (393397) „Revue des Études Augustiniennes" 18 (1972) 209-218; idem, L'activité litteraire de Jérôme de 387 à 392 „Revue de Théologie et de Philosophie" 115 (1983) 247-259.

${ }^{6}$ Cfr. Epistula 108, 7, CSEL 55. 313: „media hieme”; idem, Apologia contra Rufinum III 22, SCh 303, 272: „gravissimo frigore”; P. Lardet, L'apologie de Jérôme contre Rufin. Un commentaire, Leiden 1993, 308-309; Rufinus, Apologia in Hieronymum II 15, CCL 20, 94: „Non totos triginta dies Alexandriae ubi erat Didymus commoratus est"; Hieronymus, Apologia contra Rufinum III 22. SCh 303, 272: „Inde contendi Aegyptum, lustravi monasteria Nitriae et inter sanctorum choros aspides latere perspexi”; idem, Ad Ephesios, Praefatio, PL 26, 440B: „Alexandriam perrexi, ut viderem Didymum, et ab eo in Scripturis omnibus quae habebam dubia sciscitarer". Per un resoconto del viaggio in Egitto, cfr. idem, Epistula 108, 14, CSEL 55, 324-325; cfr. H. Grützmacher, Hieronymus. Eine biographische Studie zur alten Kirchengeschichte, II, Darmstadt 1969, 5-17; F. Cavallera, Saint Jérôme. Sa vie ef son oeuvre, I, Louvain-Paris 1922, 126-127, ibidem II, pp. 127 129; J.N.D. Kelly, Jerome. His life. Writings and Controversies, London 1975, 124-125.

${ }^{7}$ Cfr. Hieronymus, De viris illustribus 135,5 , PL 23, 719A: „Epistularum autem ad Paulam el Eustochium, quia cotidie scribuntur, incertus est numerus".

${ }^{8}$ Cfr. Saint Jérôme, Lettres, II, Paris 1951, 100. n. 2; San Girolamo, Le lettere, I, Roma 1962, 338: P. Nautin, La lettre de Paule et Eustochium à Marcelle, p. 441; P. Maraval, Saint Jérôme et le 
si potrebbero muovere delle critiche; non si sa infatti su cosa sia basata l'asserzione del copista, il quale, non conoscendo altre lettere delle pie donne e avendo sott'occhio quelle di Girolamo, potrebbe essere stato indotto a mettere una nota di chiarimento per giustificare l'anomala intestazione.

Desta poi particolare stupore una serie di elementi che contrastano sia con il gusto sia con la dottrina di Girolamo. Senza dare peso all'errore di avere scambiato il torrente Endor con il fiume Quishôn - una svista di cui però Girolamo non si accorse - è un fatto che la lettera 46 presenta scarsissimi richiami ai classici; non c'è traccia di ricercatezza letteraria ${ }^{9}$, semmai solo di erudizione biblica, con uno sfoggio di etimologie di provenienza origeniana e, indirettamente, filoniana ${ }^{10}$, che sono il più evidente contributo di Girolamo alla lettera, anzi la prova certa della sua paternità. Tutto ciò può trovare una spiegazione nel fatto che a Marcella non interessavano i classici, ma le sacre scritture analizzate a partire dal loro significato letterale ${ }^{11}$.

Suscita tuttavia maggiore perplessità, al di là del problema attributivo che può risolversi alla luce del solo esame stilistico, il fatto che sia stata sostenuta $\mathrm{e}$ poi rigettata con feroce ironia nel giro di pochi mesi, dalla primavera all'estate del 386, la leggenda di Adamo sepolto nel Golgota e purificato dal sangue di Cristo colato dalla croce. Evidentemente a Girolamo non era affatto piaciuto che si fosse interpretato il passo di Eph. 5, 14 pubblicamente, nella basilica dell'Anastasis, connettendolo con una leggenda proveniente dalla comunità giudeo-cristiana di Gerusalemme; ma Paola ed Eustochio ne furono colpite e pensarono che Marcella avrebbe apprezzato il valore antiquario della notizia e la novità nello sviluppo esegetico del passo paolino. Colpisce infine l'insistenza con cui entrambe si scusano per il disordine della loro esposizione esegetica ${ }^{12}$, che giustificano non con l'ignoranza delle regole del commento biblico, ma con il trasporto del sentimento di affetto nei confronti di Marcella ${ }^{13}$; una scusa che

pélerinage aux lieux saints de Palestine, in: Jérôme entre l'Orient et l'Occident, Paris 1988, 346, n. 8. Diversamente cfr. A. Penna, Principi e carattere dell'esegesi di san Gerolano, Roma 1950, 152, n. 1: "La lettera fu scritta da Paola ed Eustochio, ma è fuor di dubbio che essa riproduce, specialmente in questioni esegetiche, il pensiero del loro maestro".

${ }^{9}$ Cfr. H. Hagendhal, Latin Fathers and the Classics, Göteborg 1958, 97-99, 115-141, pone proprio il 386 come data d'inizio del ritorno di Girolamo allo studio dei classici; ciò non toglie che egli non avesse anche prima una prosa elengante, affettata, manierata (genus floridum), come osserva J. Fontaine, L'esthétique littéraire de la prose de Jérôme jusqu'à son second départ en Orient, in: Jérônte entre l'Orient et l'Occident, Paris 1988, 330.

${ }^{10}$ Cfr. D. Brown, Vir trilinguis. A study in the biblical exegesis of Saint Jerome, Kampen 1992 , 13-20.

11 Riferendosi all'epistolario in generale H. Hagendhal (Latin Fathers and the Classics, p. 98), afferma che „Jerome adapted his style [...] to the mind and the culture of those to whom he wrote".

${ }^{12}$ Cfr. idem, Epistula 46, 3, CSEL 54, 331: ,Turbidus sermo [...] ut multo inordinatius aliquid proferamus"; ibidem 11, p. 341: „infirmo sermone”; cfr. Eugippius, Epistula ad Paschasium 2, SCh 374, 148: „obscura disertitudine”.

${ }^{13}$ Cfr. Hieronymus, Epistula 46, 3, CSEL 54, 331. 
poteva far comodo allo stesso Girolamo, dato che la lettera, come appare inequivocabilmente dall'analisi critica, fu dettata in fretta e appena riletta per ricevere l'approvazione delle committenti per essere subito spedita a Roma con la riapertura della navigazione regolare in primavera.

Riguardo al genere, il testo appartiene a quello delle lettere dottrinali ${ }^{14}$, ben lontano dunque dalla missiva privata. Lungi dal confondere il tono di affetto nei confronti della destinataria solo come un'effusione sentimentale, esso rispecchia le formalità delle relazioni fra i membri dell'aristocrazia romana e va interpretato come uno dei luoghi comuni del bon ton di quella classe di donne elette ${ }^{15}$. La lettera 46 era destinata a Marcella e al suo circolo ascetico, ma indirettamente al pubblico cristiano di Roma; era naturale che venisse letta e divulgata tramite ricopiatura; non a caso Paolino di Nola ne venne a conoscenza. Dall'analisi retorica dell'impianto epistolare ${ }^{16}$, si deduce chiaramente che la lettera riproduce in forma scritta un tipo di dibattito che avveniva nel circolo dell'Aventino, dove Marcella conduceva l'esegesi delle Scritture con diverse allieve e dove Girolamo, durante gli ultimi anni del suo apostolato romano (382-385), aveva commentato i Salmi in progressione numerica ${ }^{17}$.

${ }^{14}$ G. Scarpat, L'epistolografia, in: Introduzione allo studio della cultura classica, Milano 1972, 493-494.

15 F.E. Consolino, Modelli di comportamento e modi di santificazione per l'aristocrazia femminile d'Occidente, in: A. Giardina (a cura di), Società romana e impero tardoantico, I. Istituzioni, ceti, economie, Bari 1986, 273-306.

${ }^{16}$ Secondo il seguente prospetto: EXORDIUM (1): Paola ed Eustochio, usando il tema retorico della impatientia caritatis cercano il consenso di Marcella, amica e maestra, alla quale si apprestano a fare una lezione di esegesi. PROPOSITIO (2): secondo Gen. 12, 1 Gerusalemme è la terra promessa. DISPOSITIO (3): si segue l'ordo artificialis. ARGUMENTATIO (4-12): prima quaestio (4) supposta che Paola e Eustochio, alias Girolamo, ipotizzano da parte di Marcella: Gerusalemme fu terra santa prima dell'uccisione di Cristo, in seguito tutta la sacralità giudaica si trasferì presso i Gentili; prima refuratio (5): il Signore, così come fece con Lazzaro, pianse Gerusalemme perché l'amava, perciò ogni cristiano deve rendere omaggio al Santo Sepolcro; seconda quaestio $(6-6,8)$ : in: Apc. 11,8 la grande città in cui fu crocifisso il Signore è chiamata spiritualmente Sodoma e Egitto, dunque Gerusalemme è Sodoma e Egitto; seconda refutatio (6, 87): in virtù del principio di non contraddizione delle Scritture e dell'interpretazione spirituale di ogni singolo passo del testo sacro, Gerusalemme non è Sodoma e Egitto, che rappresentano invece "questo mondo", Gerusalemme è la città santa; terza quaestio $(8,1)$ implicita alle finte argomentazioni polemiche precedenti: vengono chiamati in causa coloro che dicono maledetta la Giudea terrena; terza refutatio $(8,2-11)$ : i martyria di Pietro e Paolo e a maggior ragione quello di Cristo sono luoghi venerandi, solo il pellegrinaggio consente di raggiungere il grado massimo di conoscenza delle Scritture, la Palestiana è la dimora ideale del monaco, elogio di Gerusalemme e di Betlemme; contra refutatio (12): si annuncia profeticamente la caduta di Roma della quale parla $A p c .18$ chiamandola Babilonia, ancora un elogio di Betlemme; conclusio (13): ripresa del tema iniziale della impatientia caritatis, invito a Marcella perché compia un viaggio in Palestina, breve rassegna dei luoghi santi, finale con il richiamo a Cant. 3, 4 come risposta a Gen. 12, 1: Paola e Eustochio invitano Marcella a celebrare le nozze mistiche in terra santa.

17 Non si sa bene però con quale metodo e frequenza, cfr. D. Gorce, La lectio divina nell'ambiente ascetico di san Girolamo, trad. it.. Bologna 1991, 217-232. 
Fin dall'esordio Paola ed Eustochio dichiarano di volere invertire i ruoli: esse, che prima erano timide allieve e come pulcini stavano sotto le ali della chioccia, ora si sentono pronte a spiccare il volo da sole e a sciogliere il legame di dipendenza dalla maestra dell'Aventino. Rivolgendosi all'amica con il suo stesso tono didattico, la invitano in terra santa con le parole di Gen. 12, 1, cioè con quel manifesto di propaganda monastica che Girolamo aveva divulgato nel circolo dell'Aventino. Lo scopo della lettera era di dimostrare con argomentazioni esegetiche e retoriche e con la testimonianza diretta del pellegrinaggio appena compiuto, che la terra santa non solo non era maledetta a causa dell'uccisione di Gesù - come volevano certi critici malevoli provenienti, a quanto è dato supporre, dagli ambienti del clero romano più ostili alla tradizione giudaica - ma era la patria ideale di quei cristiani che volevano abbracciare la vita monastica e progredire nella conoscenza delle Scritture sulla via della perfezione. Il culto delle memorie bibliche attraverso il pellegrinaggio offriva come benefici un rinnovato sentimento di pietà e di devozione, una conoscenza approfondita e incomparabilmente più esatta dei testi sacri, una virtù cristiana più prossima allo stato di grazia e alla santità ${ }^{18}$.

Quanto agli interessi antiquari delle due pellegrine, non c'è ragione di ritenere che abbiano preferito le memorie veterotestamentarie a quelle più recenti ${ }^{19}$. Nonostante gli interessi di Girolamo fossero rivolti ai tempi remoti e oscuri delle vicende bibliche, per far luce su tutto l'insieme della tradizione sacra, la fede ardente non ha mai sviato il proposito delle due nobildonne; il ritorno costante, anche in questa lettera, del tema delle nozze mistiche del Cantico ci rassicura sull'intenso desiderio di ascetismo e di comunione mistica provato da Paola e dalla vergine Eustochio.

Dal punto di vista stilistico, la lettera appare con tutta evidenza come un esempio del trasformismo letterario di Girolamo ${ }^{20}$, al quale critici e avversari hanno spesso imputato il plagio, specie in materia di esegesi biblica; i suoi rivali lo hanno invidiato e temuto, talvolta coprendolo di accuse infamanti, a causa dei suoi rapporti privilegiati con le nobildonne romane. Non deve apparire così strano che egli abbia messo la sua formidabile penna al servizio di Paola e Eustochio; la colta Marcella non poteva che gioirne in cuore suo, dopo un anno di silenzio da parte del suo venerato maestro.

${ }^{18}$ P. Maraval, Saint Jérôme et le pélerinage aux lieux saints de Palestine, p. 348 passim.

${ }^{19}$ L'insinuazione di J. W. Smit nasce da un'irragionevole interpretazione dell'Epistula 108 , 13, CSEL 55, 323: „[Paula] cito itinere percucurrit Nazaram, nutriculam domini”, cfr. Vita di Martino. Vita di llarione. In memoria di Paola, Milano 1975, 347; i luoghi da visitare erano molti e il tempo era poco, perciò Girolamo, Paola ed Eustochio compirono il pellegrinaggio a tappe forzate.

${ }^{20}$ Per un caso analogo a questo vedi la lettera scritta a una donna dall'asceta iberico Bacharius sotto il falso nome di Marcella in PLS I 1035-1044 e il commento di G. Morin "Revue Biblique" 40 (1928) 288 passim. 


\title{
COMMENTARIO ${ }^{2 !}$
}

I.

\section{Textus epistulae}

Cp. 1, CSEL 54, 329-330:

\begin{abstract}
„Mensuram caritas non habet et impatientia nescit modum et desiderium non sustinet. Unde et nos oblitae virium nostrarum et non quid possimus, sed, quid velimus, tantum cogitantes magistram cupimus docere discipulae et, ut est vulgare proverbium: sus artium reppertricem. Tu, quae prima scintillam nostro fomiti subiecisti, quae ad hoc studium nos et sermone hortata es et exemplo et quasi gallina congregasti sub alas pullos tuos, nunc nos libere absque matre volitare pateris et accipitris pavere formidinem et ad omnem umbram praetervolantium avium formidare? Igitur, quod solum absentes facere possumus, querulas fundimus preces et desiderium nostrum non tam fletibus quam heiulatibus contestamur, ut Marcellam nostram nobis reddas et illam mitem, illam suavem, illam omni melle et dulcedine dulciorem non patiaris apud eas esse rigidum et tristem rugare frontem, quas adfabilitate sua ad simile vitae studium provocavit".
\end{abstract}

- Caritas. La lettera esordisce con un enunciato sull'amore fraterno che, visto in rapporto con il pensiero paolino di 1 Cor. 13, 4-8 è iperbolico, poiché non solo dichiara la mancanza di misura dell'amore per il prossimo, ma sembra perfino volere incrinare la fermezza del controllo del desiderio, accostando con una particella copulativa dal valore comparativo caritas a impatientia, per creare un effetto di straniamento ${ }^{22}$. A confermare la sofisticata valenza retorica dell'esordio, che presenta variazioni anche nella posizione logica di soggetto, verbo e complemento, valga un confronto con altri enunciati sull'amore presenti fin dall'inizio dell'epistolario geronimiano tutti ordinati invece secondo il pensiero paolino: ,"caritas omnia potest" ${ }^{, 23}$; ,caritas non potest comparari, dilectio pretium non habet”24; „caritas omnia sustinet" ${ }^{25}$; „caritas omnia superat et pro-

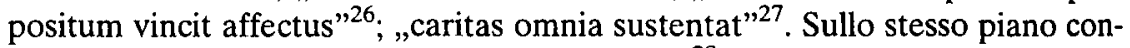
cettuale si pone invece ,amor ordinem nescit”28 che è un chiaro riferimento a Virgilio (Buc. 10, 69): „omnia vincit amor, et nos cedamus amori”. Caritas

21 Per i riscontri lessicografici e grammaticali ogni rimando è d'obbligo a $\mathrm{H}$. Gœlzer, Etude lexicographique et grammaticale de la latinité de saint Jérôme, Paris 1884.

${ }^{22}$ Sull'approccio ai temi paolini ha indagato con acume L. Perrone, Questioni paoline nell'epistolario di Gerolamo, in: Moreschini - G. Menestrina (a cura di), Motivi letterari ed esegetici in Gerolamo, Brescia 1997, 97, che ha osservato come in Girolamo ,l'elemento di originalità, più che nei contenuti in sé, va quindi cercato essenzialmente nelle forme dell'appropriazione"

${ }^{23}$ Epistula 1, 2, CSEL 54, 2.

24 Epistula 3, 6, CSEL 54, 18.

25 Epistula 7, 4, CSEL 54, 29.

26 Epistula 17, 1, CSEL 54, 70.

27 Vita Pauli 10, PL 23, 25B.

${ }_{28}$ Epistula 7, 6, CSEL 54, 31. 


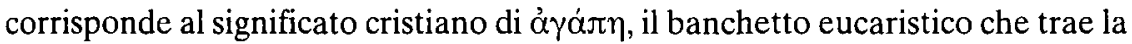
sua origine dall'usanza dei primi cristiani di pranzare in comune. Secondo Marco $(12,28)$ e Matteo $(22,40)$ „Gesù ha riassunto in due precetti il senso

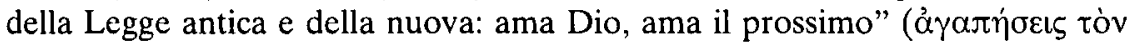

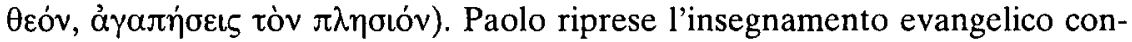
centrandosi sull'amore fraterno per il prossimo che divenne una norma fondamentale della vita cristiana ${ }^{29}$. Caritas è un concetto chiave nella conversione monastica delle due nobildonne, che hanno assunto l'insegnamento paolino come norma fondamentale della loro vita. Nell'amore per Dio e per il prossimo entrambe sentono vibrare il senso profondo dell'unione della Legge antica e nuova ${ }^{30}$. Ma non va sottovalutato il temperamento, al tempo stesso passionale e patetico, di Paola, che la rende incline a un'attitudine mistica, cosa di cui Girolamo si accorse fin dall'inizio del suo magistero romano, predisponendo per lei un'esegesi spirituale. Nel 404 Girolamo, ricordando la partenza da Roma per l'Oriente più di vent'anni prima dei vescovi Paolino di Antiochia ed Epifanio di Salamina, disse di Paola: „Non domus, non liberorum, non familiae, non possessionum, non alicuius rei, quae ad saeculum pertinet, memor sola - si dici potest - et incomitata ad heremum Antoniorum atque Paolorum pergere gestiebat [...] voto cum eis et desiderio navigabat" ${ }^{\text {, }}$ - il verbo gestire ${ }^{32}$ rivela in modo realistico il vero temperamento di Paola, tanto da rendere credibilissima la vivacità e l'entusiasmo del suo carattere descritti da Girolamo $^{33}$. Non solo Paola si fece coinvolgere negli ambiziosi progetti di Girolamo, che a Betlemme portò a compimento la Vulgata e i commenti biblici, ma riuscì a convincere anche la figlia a condurre una vita cenobitica lontano da Roma. Ostentatamente immemori della virtù della sopportazione $\mathrm{e}^{34}$, anzi, ben esercitate sul tema dell'impatientia caritatis ricorrente nelle declamazioni pseudoquintilianee ${ }^{35}$, Paola ed Eustochio, ovvero Girolamo, rendono

${ }^{29}$ Cfr. E. Stauffer, Grande lessico del Nuovo Testamento [d'ora in poi GLNT], I, Brescia 1965, 92-146, s.v. áyaráw.

${ }^{30}$ Cfr. Hieronymus, Ad Galatas 3, 5, PL 26, 409A: „Tantum autem bonum est caritas, ut omnis Lex in illa recapituletur".

31 Epistula 108, 6, CSEL 55, 311.

32 Il verbo ricorre anche in ibidem 28, CSEL 55, 347, riferito all'anima di Paola ,smaniosa" (gestiens) di uscire dal corpo per raggiungere Dio.

${ }^{33}$ Non mi pare sostenibile l'affermazione di F.E. Consolino, Modelli di comportamento e modi di santificazione per l'aristocrazia femminile d'Occidente, p. 294: „le diversità dovute alla differenza di temperamento non possono essere considerate significative ai fini di una ricostruzione storica".

${ }^{34}$ Tertulliano e poi Cipriano, sulla scorta di una tradizione letteraria anteriore di matrice stoica, vi avevano dedicato due operette: rispettivamente il De patientia, SCh 310 , e il De bono patientiae, PL 4, 645-662.

${ }^{35}$ Cfr. Declamationes V 3, 10, dove si parla di una "Inaequalitate caritatis" del padre; ibidem V 3, 16: „Impatientia est hominis qui magis ametur; ibidem VIII 4, 23-24: „Vultis intellegere, iudices, nihil impatientia caritatis fecisse patrem?”; ibidem XVIII 5, 16-17: „Sane faciat vos pronos ad suspiciones nimia caritas, et [ex] impatientia diligendi plerumque descendat, ut credas facile, 
omaggio a Marcella secondo una consuetudine che della costanza del legame affettivo, quando non addirittura passionale, tra maestro e allievo, faceva il perno dell'educazione antica ${ }^{36}$. Ma sulla madre e sulla figlia agì come forte stimolo l'interpretazione ascetica data al concetto di caritas da Origene, così come potevano leggere nella versione latina di Girolamo: „Caritas non sinit permanere in te vallem quod, si habueris et patientiam et bonitatem, non solum vallis esse desistis, sed etiam mons esse incipies Dei" ${ }^{37}$.

- Unde... discipulae. Il discorso, non del tutto stereotipato, rientra nell'economia retorica dell'esordio epistolare. Paola ed Eustochio fingono di non potere sostenere il ruolo di magistrae nei confronti di Marcella. Ma di nuovo l'entusiasmo e un pizzico di salace ironia - altra prova evidente della paternità geronimiana della lettera - vengono in loro soccorso. Paola in realtà era una donna coraggiosa e aveva carattere, come dimostrò non solo nel momento in cui prese la decisione di lasciare Roma, ma subito dopo nell'affrontare il pellegrinaggio in condizioni molto difficili. Che volesse impartire con la figlia una lezione a Marcella, era la dimostrazione palese del suo definitivo affrancamento dal circolo dell'Aventino. Marcella era puntigliosa ed esigente nel commento alle Scritture; ma ora le sue ex discepole si sentivano finalmente sicure, perché credevano di avere acquisito un grado di conoscenza superiore grazie alla visione diretta dei luoghi santi; sicché Girolamo, parafrasando Ovidio, poteva dire di loro che bastava il desiderio a renderle eloquenti ${ }^{38}$.

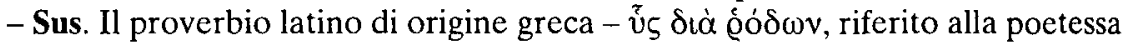
Corinna -, ricorre anche in $E p .58,7$ senza l'uso dell'antonomasia. Girolamo lo mutuò da Cicerone (Acad. 5 e Orat. II, 57).

- Docere. Assunto nella sua valenza retorica è pari all'influenza intellettuale che l'oratore intende esercitare sull'arbitro della situazione ${ }^{39}$. Marcella, ligia per educazione alla modestia delle matrone romane e fedele all'umiltà monastica secondo il divieto dell'apostolo Paolo di insegnare alle donne, era piena di scrupoli e non voleva ostentare la sua erudizione. Spesso, anzi, esprimeva opinioni sue facendole passare per quelle di Girolamo: „Sic interrogata respondebat, ut etiam sua non sua diceret, sed vel mea, vel cuius libet alterius, ut et in ipso quod docebat, sed discipulam fateretur. Sciebam enim dictum ab apostolo: docere autem mulieri non permitto [1Tim. 2, 12]"40. Passata al vaglio di Girolamo, la lectio divina giunse sotto forma di lettera dottrinale alla maestra dell'Aventino.

quod timeas". Che Girolamo conoscesse bene questi testi scolatici è prova il suo riferimento diretto in Epistula 36, 14, CSEL 54, 280: „Quintiliani flosculis et scolari declamatione”; cfr. H. Hagendhal, Latin Fathers and the Classics, pp. 296-297, 312.

36 Cfr. H.I. Marrou, Histoire de l'éducation dans l'antiquité, I, Paris 1948, 63-64.

37 Origenes, In Lucam hom. 22, 1, SCh 87, 300, la traduzione di Girolamo risale al 392.

38 Cfr. Ovidius, Ars amandi I 610: „Fac tantum cupias, sponte disertus eris”.

${ }^{39}$ Cfr. H. Lausberg, Elementi di retorica, trad. it., Bologna 1969. 51.

40 Epistula 127, 7, CSEL 56, 151; cfr. 1Tim. 2, 12. 
- Nos... formidare. Similitudine di lontana eco omerica (Ilias XXII 139): „,come quando sui monti un falco - il più veloce fra gli uccelli piomba di slancio su una colomba tremante" ${ }^{41}$. I versi omerici si riferiscono ad Achille che insegue Ettore. Ovidio (Ars amandi I 117) si appropriò dell'immagine sostituendo il falco con le aquile. Paola ed Eustochio, paragonandosi ai pulcini, amplificano la figura retorica citando oltre al falco, genericamente, tutti gli uccelli rapaci; esse assumono tuttavia il punto di vista delle inseguite da terra; anche Gregorio di Nazianzo aveva paragonato il giovane Basilio a un pollo che zampetta dietro alla madre ${ }^{42}$. Come variante della metafora nautica praetervolare "spiccare il volo", equivale a „spiegare le vele” „accingersi a scrivere"43. L'espressione è topica nell'esordio, specialmente nei testi poetici, e in questo caso esprime il desiderio delle pie donne di staccarsi dall'autorità guida di Marcella per procedere da sole nell'interpretazione delle Scritture ${ }^{44}$. Proprio per questo non $\mathrm{mi}$ pare che si possa sostenere la tesi della „inferiorità" intellettuale di Paola e della figlia rispetto alle altre nobildonne cristiane di Roma, discepole e corrispondenti di Girolamo, né tantomento insinuare che la loro „docilità" nei confronti di Girolamo sia dovuta a una mancanza di carattere ${ }^{45}$. La scelta di Paola di abbandonare Roma e la famiglia e quella della giovane Eustochio di rimanere vergine e di consacrarsi per sempre a Dio a Betlemme presso la basilica della Natività, lasciano intendere il grande spirito che animava le due pie donne, certamente sollecitate a un proposito così eroico da colui che più di ogni altro ha promosso il monachesimo in Occidente nel secolo IV.

- Marcellam nostram. Marcella, prima definita maestra, poi paragonata alla chioccia che raccoglie i pulcini sotto le sue lil $^{46}$, è ritratta dal vivo, con insistita anafora asindetica, come una persona mite, soave, dolce più del miele e della dolcezza stessa. Ma l'ultima immagine, che la dipinge affabile, è quella che meglio si adatta a restituirci la sua personalità. La più grande passione di Marcella era di parlare, discutere e coinvolgere le amiche discepole nelle sue riflessioni intellettuali e nelle sue sacre meditazioni; è questo il ruolo principale che Paola ed Eustochio le riconoscono, debitrici della loro scelta di vita ascetica. Che Marcella fosse a conoscenza delle Scritture al punto di poter eserci-

${ }^{41}$ Omero, Iliade, trad. it. M.G. Ciani, Torino 1998, 959.

${ }^{42}$ Cfr. Oratio 43, 12, SCh 384, 142.

${ }^{43}$ Il participio praetervolantium ricorre diversamente anche in Hieronymus, Vita Hilarionis 20, PL 23, 40.

${ }^{44}$ Cfr. E.R. Curtius, Letteratura europea e Medio Evo latino, a cura di R. Antonelli, Firenze 1992, 147-150.

${ }_{45} \mathrm{Per}$ un giudizio positivo su Marcella, ma limitativo sulle capacità intellettuali di Paola e Eustochio, mitigato tuttavia anche da qualche ripensamento nelle note, cfr. F.E. Consolino, Modelli di comportamento e modi di santificazione per l'aristocrazia femminile d'Occidente, pp. 393394, nn. 181-184.

${ }^{46}$ L'immagine è anche in Mt. 23, 38. Questa espressione è tipica della Palestina cfr. E. Lohse,

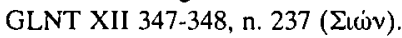


tare un vero ruolo di magistero nel circolo femminile cristiano dell'Aventino, è provato in maniera esplicita da questa lettera e da altre testimonianze di stima e di valore da parte di Girolamo ${ }^{47}$. Egli scrisse ai posteri che in Marcella

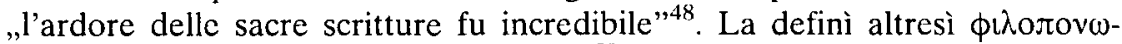

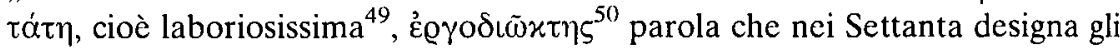
Egiziani incaricati di sorvegliare il lavoro degli Ebrei: essi li bastonano senza riguardo perché portino a termine il loro lavoro ogni giorno ${ }^{51}$. Con lo stesso epiteto Origene aveva chiamato il suo discepolo ed ammiratore Ambrogio, che lo spingeva senza posa al lavoro come ,le chef de corvée de Dieu"52. Quando, lontano da Roma, Girolamo non poté più esercitare direttamente il suo magistero, non trovò di meglio che raccomandare la saggia Marcella e la sorella Asella alla giovane vergine Principia intenzionata ad abbracciare la vita ascetica per meglio vivere il senso delle Scritture ${ }^{53}$. Dopo la partenza di Girolamo, Marcella era diventata a Roma il punto di riferimento più autorevole per dirimere i quesiti sulle Scritture e veniva consultata "come un vero oracolo, come un portavoce autorizzato" di Girolamo ${ }^{54}$; il quale aveva perfino detto di lei: „Nec sine ratione praeiudicata apud eam valebat auctoritas, sed existimabat omnia, et sagaci mente universa pensabat, ut me sentirem non tam discipulam habere quam iudicem"55. Marcella restava pur sempre un „giudice" e dato il

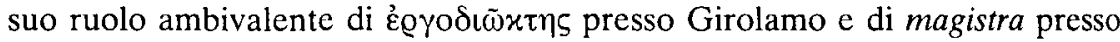
Paola ed Eustochio, tanto maggiore fu il loro sforzo persuasivo.

- Mitem... suavem. Il ritratto di Marcella mite, soave e affabile è altresì un ritratto del suo gusto stilistico, che riflette in parte quello dell'aristocrazia colta di Roma e che ha il suo fondamento teorico in Quintiliano ${ }^{56}$. Girolamo sottolinea la nobiltà del gusto oratorio dell'amica - mancano infatti riferimenti

\footnotetext{
${ }^{47}$ Cfr. Epistula 127, 5, CSEL 56, 149: „Qualis magistra... talis discipilae”.

${ }^{48}$ Cfr. Epistula 127, 4, CSEL 56, 148: „Divinarum scripturarum ardor incredibilis”.

${ }^{49}$ Cfr. Epistula 30, 14, CSEL 54, 249.

${ }^{50}$ Crr. Epistula 28, 1, CSEL 54, 227, così è definito anche Nepoziano nel suo elogio funebre
} cfr. Epistula 60, 1, CSEL 54, 549.

5i Cfr. Exodus 3,$7 ; 5,6 ; 10 ; 13$.

52 In loannem V Proemium, SCh 120, 372; Hieronymus, De viris illustribus 61, PL 23, 671-673; P. Jay, L'exégèse de saint Jérôme, Paris 1985, 51, n. 160.

53 Epistula 65, 2, CSEL 54, 618-619: „ita ut post profectionem nostram, si aliquo testimonio scripturarum esset aborta contentio, ad illam iudicem pergeretur".

${ }^{54}$ Cfr. Epistula 127, 7, CSEL 56, 151; D. Gorce, La lectio divina nell'ambiente ascetico di san Girolamo, p. 240.

5s Ad Galatas, Praefatio, PL 26, 307-309. Aristotele (Rhetorica II B 18, 1391 b), sostiene che „anche se l'uditore è uno solo, nondimeno è pur giudice. poiché, in generale, colui che si deve persuadere è un giudice".

${ }^{56}$ Cfr. Institutio oratoria II 8, 4: „Generi dicendi [...] dulci”. Cfr. Thesaurus Linguae Latinae [d'ora in poi TLL] V 1, 2190-2195, s.v. dulcis; già Cicerone, Orator 19, 62, pur distinguendo il discorso dei grandi filosofi e oratori greci da quello latino forense, aveva definito Platone principe dell'eloquenza „et suavitate et gravitate”. Tuttavia per un significato negativo dato ai dulces 
all'aspetto fisico e ai tratti somatici - usando espressioni d'affetto che intorno alla metà del secolo IV dalla sfera dell'intimità erano passate al formulario cerimoniale. Il gusto di Marcella, donna equilibrata e aliena dalle passioni, assai diversa per temperamento sia da Girolamo che da Paola e Eustochio, si chiarisce analizzando i riferimenti che Girolamo fece su di lei nei suoi scritti. In tal senso i rimproveri di Marcella agli eccessi polemici del suo maestro vanno considerati anche come una critica alla sua spregiudicatezza stilistica. E' noto l'imbarazzo con cui Marcella e Pammachio dovettero difendere i libelli più feroci provenienti dallo scriptorium di Betlemme. Girolamo non rinunciò a contenersi nei limiti del sermo humilis ${ }^{57}$. Il suo desiderio, una volta resa inossidabile la verità della littera mediante l'impiego di ogni rigore filologico ed esegetico, fu quello di innalzare la lingua dei testi sacri al sublime. Dove poté, Girolamo spinse il latino della Vulgata verso un nuovo genere di pathos, specie nelle sue rielaborazioni letterarie esterne ai commentari e in particolare nell'epistolario. In una visione così rinnovata dello stile e della cultura tardoantica, egli finì per sostituire il modello degli eroi antichi con i profeti, con gli apostoli e con Cristo.

- Omni melle. L'espressione è ripresa da Plauto (Asinaria 614): „Oh! melle dulci dulcior mihi tu es". Il paragone con il miele acquista maggior rilievo in virtù della figura etimologica. Il miele e le api sono simboli della poesia $\mathrm{e}$ dell'eloquenza da Omero agli autori cristiani; dice infatti Platone (lone 534 A-B): „I poeti ci dicono che attingono i loro canti da fonti che versano miele e da giardini e da boschetti che sono sacri alle Muse, e che a noi riportano, anch'essi volando come le api" 58 . Paolino narra nella Vita di Ambrogio che uno sciame prodigioso di api „coprì il volto e la bocca" del santo ancora in fasce nella culla, preannunciando in tal modo la grandezza della sua eloquenza; e subito dopo cita Prov. 16, 24 , i buoni discorsi sono favi di miele" ${ }^{\text {. }}$. Frequente la metafora del miele in poesia: ,musaeo melle" (Lucretius, I $947=$ IV, 22); „poetica mella” (Horatius, Ep. I 19, 24); „Heliconia mella” (Claudianus, Laus Serenae, proem.). Basilio di Cesarea interpreta le api come un esempio di saggezza ed assume il miele come metafora del bene: „le api a differenza degli altri animali che si limitano al godimento del profumo e del colore dei fiori, sanno trarre da essi anche il miele" $" 60$.

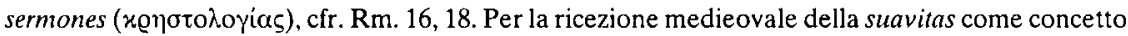
stilistico cfr. E.R. Curtius, Letteratura europea e Medio Evo latino, pp. 133, 396, n. 24.

${ }^{57}$ Cfr. E. Auerbach, Studi su Dante, trad. it., Milano 1984, 167-175 „Sacrae Scripturae sermo humilis".

${ }_{58}$ Platone, Tutti gli scritti, trad. it. di G. Reale, Milano 1991, 1027.

59 Vita Ambrosii 3, in: Vita di Cipriano, Vita di Ambrogio, Vita di Agostino, a cura di A.A.R. Bastiansen, Milano 1975, 58.

6) Oratio ad adulescentes 4, 8, trad. it. a cura di M. Naldini: Basilio di Cesarea, Discorso ai giovani, Firenze 1984, 93-94.
} 
- Rugare frontem. L'espressione deriva dal lessico giudiziario ${ }^{61}$. Ammiano Marcellino (XXX 4, 19) fece un ritratto ironico e caricaturale degli oratori forensi di bassa lega suoi contemporanei ${ }^{62}$. Allo stesso modo Girolamo descrisse l'atteggiamento dello stenografo spazientito e contrariato dalle sue pause meditative: „Tunc me tacitus reprehendat, manum contrahit, frontem rugat"63. Marcella dovette più volte corrugare la fronte, non solo quando si atteggiava a moderatrice tra le giovane discepole, ma soprattutto quando Girolamo era in collera. Egli così le scriveva: „Scio te cum ista legeris, rugare frontem et libertatem rursum seminarium timere rixarum ac meum, si fieri potest, os digito velle comprimere, ne audeam dicere quae alii facere non erubescunt" ${ }^{64}$; Marcella in fondo esercitava su Girolamo una benefica moderazione. Del resto, sui sentimenti di affetto e di stima reciproci, durante il soggiorno di Girolamo sull'Aventino, non ci sono dubbi. Così si esprimeva Girolamo nei confronti della madre di Marcella: „Albinam comunem matrem valere cupio [...] eamque per te salutari obsecro et duplicis pietatis officio focilari, quo in una atque eadem christiana simul diligatur et mater"65.

- Adfabilitate. L'affabilità è una caratteristica del monaco che ha trasformato le sue parole e i suoi gesti in un unico strumento al servizio di Dio. Tra le varie figure dei monaci orientali, può essere preso ad esempio l'abbâ Bès ricordato

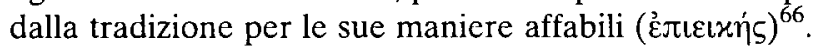

- Studium. Il termine è usato come variante di ,propositum" e „conversatio

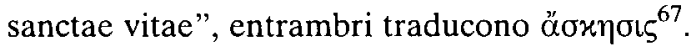

Cp. 2, CSEL 54, 330-331:

„Certe, si sunt meliora, quae poscimus, non est impudens desiderium. Si cunctae scripturarum voces nostrae sententiae congruunt, non faciamus audacter ad ea te provocantes, ad quae tu nos saepissime cohortata es. Prima vox dei ad Abraham: exi, inquit, de terra tua et de cognatione tua et vade in terram quam monstrabo tibi. Iubetur patriarchae, ad quem primum de Christo est facta promissio, ut relinquat Chaldaeos, relinquat confusionis urbem et Roboth, id est latitudines eius, relinquat campum Sennaar, in quo superbiae usque ad caelum erecta turris est, et post fluctus istius saeculi, post flumina, super quae sederunt sancti et fleverunt, cum

${ }^{61}$ Cfr. Seneca Rhetor, Controversia 10, praefatio 9: „Licet Mela meus contrahat frontem”: Seneca, De beneficiis 1,$1 ; 7,1$ : „Colligit rugas et trahit frontem”.

62 Cfr. P. Antin, Recueil sur saint Jérôme, Bruxelles 1968, 213.

63 Ad Galatas III, Incipit, PL 26, 399-400.

64 Epistula 27, 2, CSEL 54, 224-225; F. Cavallera, Saint Jérôme. Sa vie et son oeuvre, I, Louvain -Paris 1922, 86, n. 3.

65 Epistula 32, 2, CSEL 54, 252-253.

${ }^{66}$ Cfr. Historia monachorum in Aegypto 4, in: A.J. Festugière, Les moines d'Orient, IV, 1. Paris 1965, 36-37.

${ }^{67}$ Cfr. Hieronymus, Vita Hilarionis 14, PL 23, 34 D; Sulpicius Severus, Vita Martini 22, SCh 133,300 . 
recordarentur Sion, post gravem gurgitem Chobar, de quo Hiezechiel capillo verticis sublevatus Hierosolyma usque transfertur, habitet terram repromissionis, quae non rigatur ut Aegyptus de deorsum, sed de sursum, nec facit holera languentium cibos, sed temporaneum et serotinum de caelo expectat imbrem. Haec terra montuosa et in sublimi sita, quantum a deliciis saeculi vacat, tantum maiores habet delicias spiritales. Denique et Maria, mater domini, postquam ad eam angeli est facta promissio et uterum suum intellexit esse domum filii dei, derelictis campestribus ad montana perrexit. De hac urbe allophylo quondam hoste superato ac diabolicae percussa frontis audacia, postquam ille in faciem conruit, exultantium animarum turba processit et concinens chorus decem milium David nostri victoriam praedicavit. In hac angelus gladium tenens et totum inpietatis devastans orbem in Orna, Iebusaeorum regis, area templum domini designavit iam tunc significans ecclesiam Christi non in Israhel, sed in gentibus consurgentem. Recurre ad Genesim, et Melchisedech, regem Salem, huius principem invenies civitatis, qui iam tunc in typo Christi panem et vinum obtulit et mysterium Chrystianum in salvatoris corpore et sanguine dedicavit".

- Si... poscimus. La Roma del tardo secolo IV pullulava di individui fortemente intenzionati a distinguersi, singolarmente o all'interno di gruppi élitari, dai loro simili e da una società „fluida e maldefinita”; perfino „l'aristocrazia era un gruppo eterogeneo e, in parte, non classificabile"68. Il senato di Simmaco si contrapponeva emblematicamente al senato dell'Aventino ${ }^{69}$. Il senatore pagano era fiero di appartenere alla „parte migliore del genere umano”, ma riconosceva, con più tolleranza dell'ortodosso Girolamo, che erano varie le strade della fede e della conoscenza ${ }^{70}$. Girolamo invece, ammoniva Eustochio ad essere „santamente orgogliosa" nella consapevolezza che, conservando la purezza virginale, sarebbe stata migliore delle altre ${ }^{71}$. Già Cipriano nel De habitu virginum aveva glorificato il coro delle vergini definendolo come „illustrior portio gregis Christi"72. Dopo circa un secolo erano i gruppi e gli individui dell'aristocrazia romana ad essere contagiati da quello stesso idealismo ascetico frenetico che prima aveva coinvolto solo il ceto medio. In quel tempo anche la coppia imperiale, Teodosio e Flaccilla, che già versava denaro nelle opere pie,

${ }^{68}$ P. Brown, Religione e società nell'età di sant'Agostino, trad. it., Torino 1975, 175-178; cfr. F. Jacques, L'ordine senatorio attraverso la crisi del III secolo, in: A. Giardina (a cura di), Societa romana e impero tardoantico. I. Istituzioni, ceti, economie, Bari 1986, 81-225.

${ }^{69}$ Cfr. Hieronymus, Epistula 97, 3, CSEL 55, 184: „Vos, christiani senatus lumina”.

${ }^{70} \mathrm{Cfr}$. Simmachus, Epistula I 52; Relatio III 10: „Uno itinere non potest perveniri ad tam grande secretum", in cui si esprime un'idea già di Platone, Phaedon $108 \mathrm{~A}$; cfr. S. Rebenich, Hieronymus und sein Kreis, Stuttgart 1992, 181-192 (Pars melior generi humani).

7 Cfr. Hieronymus, Epistula 22, 16, CSEL 54, 163: „Disce in hac parte superbiam sanctam, scito illis esse meliorem"; cfr. P. Brown, Il corpo e la società, trad. it., Torino, 1992, 334-353. Sulla fierezza come virtù principale dell'estasi cfr. Angela da Foligno, Il libro dell'esperienza, a cura di G. Pozzi, Milano 1992, 39, dove si citano i casi di Beatrice di Nazareth e di Jacopone da Todi.

72 De habitu virginum 3, PL 4, 443 B vel CSEL 3/1, 189; cfr. D. Gorce, La lectio divina nell'ambiente ascetico di san Girolamo, p. 45. 
era altresì impegnata in un incessante "sollecitudine o zelo verso qualsiasi genere di miglioramento"73. La ricerca del vero bene, oltre a un mutamento delle abitudini di vita, implicava uno sforzo di rinnovamento introspettivo. La conoscenza della propria interiorità era ottenuta attraverso l'assiduo studio delle Scritture e la meditazione della dottrina cristiana. Paola ed Eustochio si affidarono ad un precettore spirituale d'eccezione, il quale mise a disposizione non solo la sua pratica ascetica e la sua conoscenza biblica, ma fornì anche degli strumenti di verifica spirituale e di studio, come le omelie di Origene sul Cantico dei Cantici e la lettera 22, autentica istruttoria sulla verginità monastica, che riassume molti temi ascetici del grande commentario di Origene al Cantico ${ }^{74}$. - Non... desiderium. In nome del bene che vogliono ottenere, Paola ed Eustochio si difendono da un'eventuale accusa di impudenza: desiderium va qui inteso come il sentimento che le spinge a scrivere in maniera autorevole all'amica, un tempo già maestra. Grazie all'esempio di Marcella, madre e figlia hanno rinnovato la loro pudicizia con un nuovo „vitae studium”. Girolamo, che ha esaltato in senso assoluto la verginità agli occhi della giovane Eustochio, da poco convertita alla vita monastica, indugia sul valore della pudicizia intesa come scrupolosa castità di corpo e di spirito, non come mera continenza; la quale implicherebbe il matrimonio, un bene mondano ehe contrasta con la ricerca del vero bene ${ }^{75}$. Alle nobildonne cristiane che sono rimaste vedove come Paola o come, in seguito, sua nuora Leta, o addirittura che sono divorziate come Fabiola, ma sono intenzionate a condurre una vita ascetica, Girolamo prescrive con un rigore assoluto la „pudicizia eterna”, il solo mezzo che unito alla fede e alle elemosine conduce alla santità ${ }^{76}$. Nella Vita di Malco Girolamo aveva indicato la pudicizia come virtù monastica associandola all'idea del martirio: „Habet et servata pudicitia suum martirium"77. Con la stessa espressione si rivolge a Demetriade ${ }^{78}$. Anche Claudiano elogia a lungo Serena per la sua pudicizia ${ }^{79}$. L'incontenibile pienezza della caritas divina consente alla madre e alla figlia di trasformare il loro desiderio e il loro entusiasmo, che male si concilierebbero con la pudicizia tradizionale, in un'inedita prova di fede e di amore nei confronti di Dio e di Marcella ${ }^{80}$.

${ }^{73}$ Gregorius Nissenus, Oratio funebris in Flacillam, PG 46, $881 \mathrm{C}$.

${ }^{74}$ Cfr. Origenes, In Canticum Canticorum II 5, 39, SCh 375, 376 (trad. latina di Rufino): „Necesse est animam, eam praecipue, quae bona et pulchra et sensibus et ingegno vigilans, cognoscere semet ipsam et dare operam ad agnitionem sui per exercitia doctrinae et studia divina".

75 Cfr. Hieronymus, Epistula 22, 2, CSEL 54, 146.

76 Cfr. idem, Epistula 108, 26, 5, CSEL 55, 345; idem, Epistula 127, 2, CSEL 56, 146.

77 Idem, Vita Malchi 6, PL 23, 56 D.

${ }^{78}$ Cfr. idem, Epistula 130, 5, CSEL 55, 179: „Quid agis, Demetrias? cur pudicitiam tanto pavore defendis? libertare opus est et audacia, quae sic in pace metuis, qui facere in martyrio perpetrando?"

${ }^{79}$ Cfr. Claudianus, Elogium Serenae, trad. it. a cura di F.E. Consolino, Venezia 1986, 28.

${ }^{80} \mathrm{La}$ prova dell'avvenuta emancipazione è nell'apprendimento della dottrina ascetica di 
- Non... audacter. Paola ed Eustochio precisano che il loro atteggiamento non vuole essere provocatorio. La loro audacia non contrasta con la pudicizia; l'audacia infatti era disdicevole per una donna ${ }^{81}$; secondo l'etica romana tradizionale la matrona doveva mostrarsi estranea all'audacia, anzi era suo dovere moderare gli impeti del marito e dei famigli ${ }^{82}$. Le donne dell'aristocrazia erano educate rigidamente more maiorum; Girolamo ne era consapevole, perciò faceva leva sul loro orgoglio per liberarle dal giogo degli antenati ed incitarle ad una nuova coraggiosa scelta di vita, così che poteva scrivere a Demetriade: „libertate opus est et audacia" 83 .

- Exi de terra tua. Il monito di Genesim 12, 1 ha significati molteplici: letteralmente è un richiamo alla volontà di Dio; spiritualmente rappresenta la speranza di raggiungere la comunità dei perfetti, la Gerusalemme celeste; storicamente è un recidere le radici della propria stirpe e della propria terra per incamminarsi, come pellegrini verso la terra promessa; allo stesso tempo, è un invito a considerare la vita di questo mondo come un passaggio al regno di Dio; misticamente invece equivale ad un invito al „conosci te stesso" e rappresenta l'uscita dell'anima dal corpo per l'incontro con $\mathrm{Dio}^{84}$; simbolicamente raffigura l'uscita dalla terra d'Egitto e la traversata del Mar Rosso, un itinerario che, nell'insegnamento di Origene, conduceva alla terra promessa ${ }^{85}$. A Roma, Girolamo aveva diffuso nel circolo dell'Aventino il passo della Genesi come un manifesto monastico per coloro che volevano intraprendere il cammino della perfezione, in un momento in cui il modello cristiano di vita contemplativa minacciava la compattezza e la sopravvivenza delle famiglie nobili; l'aristocrazia tendeva ,, frantumarsi, a formare piccoli gruppi, ciascuno anelante ad essere un'élite, ciascuno ansioso di elevarsi al di sopra dei vicini e dei rivali" ${ }^{\prime 86}$. In tale ambiente, al fiore delle nobildonne romane, Girolamo predicava una conversione all'ascesi che „implicava un rifiuto della vita sociale dell'Urbe e, quasi inevitabilmente, l'abbandono di Roma per i centri della nuova religiosità, i luoghi santi e l'Egitto"87.

Origene cfr. In Canticum Canticorum I 4, 4, SCh 375, 222: „Trahit enim unaquaeque anima et adsumit ad se Verbum Dei pro capacitatis et fidei suae mensura".

${ }^{81}$ Cfr. Hieronymus, Vita Hilarionis 13, PL 23, $33 \mathrm{C}$ : "Ignosce... audaciae".

82 Cfr. il testo emblematico della Laudatio Turiae, in: L. Storoni Mazzolani, Una moglie, Palermo 1982, 80.

${ }^{83}$ Hieronymus, Epistula 130, 5, CSEL 56, 179.

${ }^{84}$ Cfr. Mt. 10, 37; Origenes, In Canticum Canticorum II 5, 1-40, SCh 375, 354-379; idem, SCh 376, 770-772, n. 16 (commento).

${ }^{85}$ Cfr. l'introduzione di Rousseau alle due omelie di Origene tradotte da Girolamo in SCh 37 bis, 26-37, particolarmente. Le sentenze di Origene hanno un significato propriamente mistico, non estatico, essendo la sua ,una mistica speculativa dell'illuminazione dello spirito attraverso la gnosi [...] che culmina nella contemplazione dei misteri", cfr. J.P. Daniélou, Origène. Il genio del cristianesimo, trad. it., Roma 1991, 345-362.

${ }^{86}$ P. Brown, Religione e società nell'età di sant'Agostino, p. 176.

${ }^{87}$ Ibidem, p. 157; idem, Il corpo e la società, pp. 334-353. 
Paola ed Eustochio ripeterono a Marcella la prima vox Dei ad Abraham che Girolamo, due anni prima, aveva ricordato a Paola in preda al dolore e alla disperazione per la perdita della primogenita Blesilla ${ }^{88}$. Girolamo aveva ripetuto lo stesso monito ad Eustochio all'inizio dell'Epistula 22 e alla medesima lo ribadirà al momento della morte della madre ${ }^{89}$. Egli testimonia che Paola, poco prima di morire, avrebbe pronunciato il verso 11 del Salmo 83: „scelsi d'essere negletta nella casa del mio Signore, piuttosto che abitare nelle tende dei peccatori" ${ }^{90}$. Proprio perché Paola dopo la morte del marito, nel $381^{91}$, aveva fatto sue queste parole, assumendole come un monito interiore, come un principio di conversione del cuore, Girolamo poté ripetergliele in uno dei momenti più critici della sua vita, quando il dolore incontenibile per la morte della figlia sembrò smentire tutti i buoni propositi spirituali della madre ${ }^{92}$. Ricordare ora a Marcella quel passo biblico, dalla terra promessa, significava annunciare una conquista spirituale e una guarigione definitiva dal dolore materno. Nel suo commento a Gen. 12, 1, Origene non aveva fatto alcuna allusione all'oracolo divino come monito alla conversione ascetica; si era limitato a constatare che, in quel momento Abramo era chiamato ancora Abram, non Abraham, portando il nome della sua nascita carnale", e non era ancora circonciso e non aveva ancora accolto l'alleanza (testamentum) di Dio ${ }^{93}$. Nel prologo al commentario del Cantico tuttavia, egli aveva interpretato Abramo come figura della filosofia morale: „Tanta enim fuit eius oboedentia et tanta observatio mandatorum, ut, cum audiret: exi de terra tua [...] non sit cunctatus, sed statim fecerit"94. Didimo il Cieco invece aveva commentato così l'abbandono del padre idolatra da parte di Abramo: „Non conveniva che colui che aveva la fede in Dio restasse presso gli uomini perversi [...] perché la frequentazione dei cattivi nuoce spesso agli uomini zelanti, soprattutto a quelli che lo sono da poco"; l'anima che progredisce nel suo zelo secondo la volontà di Dio, ottiene ,una grandezza che non è più terrestre ma celeste"; egli aveva esortato a "dimenticare tutte le cose terrestri”, sostenendo che la terra promessa non era una „terra visibile [...] ma invisibile"; poi aveva commentato: „Ogni peccatore ha il diavolo per padre [...] Dio gli raccomanda

${ }^{88}$ Cfr. Hieronymus, Epistula 39, 6, CSEL 54, 305-307.

${ }^{89}$ Cfr. Epistula 108, 31, CSEL 55, 349.

90 Ibidem 28, CSEL 55, 347; anche san Severino in punto di morte ammonisce i confratelli a seguire l'esempio di Abramo, cfr. Eugippius, Vita Severini 43, SCh 374, 282: „Huius igitur beati patriarchae imitamini fidem, imitamini sanctitatem, terrena dispicite, patriam celestem semper inquirite".

91 Cfr. P. Nautin, Etudes de chronologie hiéronymienne (393-397), p. 218; seguito da J.N.D. Kelly, Jerome. His life, writings and controversies, p. 92, n. 6.

92 Cfr. Hieronymus, Epistula 39, 5, CSEL 54, 304: „Cum sciam toto renuntiasse te mundo et abiectis calcatisque deliciis orationi, ieiuniis, lectioni vacare cotidie, cum ad exemplum Abraham cupias exire de terra tua et de cognatione tua, ut Chaldaeis et Mesopotamia derelictis terram repromissionis introeas".

93 Cfr. Origenes, Homilia in Genesim III 3, SCh 7 bis, 120-125.

94 Origenes, In Canticum Canticorum, Prol. 3, 18, SCh 375, 140. 
dunque di lasciare quella che egli chiama terra $(\gamma \tilde{\eta} v)$ - poiché non c'è niente di celeste (ov̉@óvov) nell'Avversario, il diavolo, ma in lui tutto è terrestre ( $\pi \dot{\alpha} v \tau \alpha$ $\gamma \eta \dot{\eta}(v)$ ) - in modo tale che essendosi spogliato dell'immagine di fango (

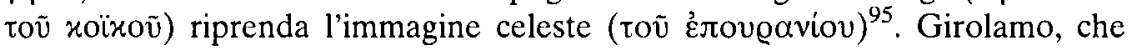
aveva approfondito lo studio del misticismo origeniano a Costantinopoli sotto la guida di Gregorio di Nanzianzo ${ }^{96}$, non aveva fatto altro che riversare sulle sue discepole romane l',,onda inebriante" (Brown) della spiritualità orientale. Insegnando a Eustochio che la verginità non è un mero ideale, ma „la condizione tipica della generazione divina e dei rapporti tra il Padre e il Figlio", Girolamo intedeva dire alla giovane aspirante asceta che il suo dovere era di „conservare la condizione originaria dell'uomo, quella creata da Dio", poiché la salvezza si

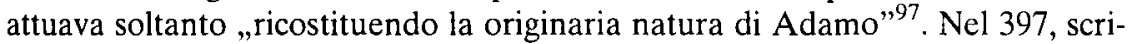
vendo a Principia sul Salmo 44, egli echeggiò le parole di Didimo nel commento ai versetti 11-13, quando scrisse che la vergine, sposa di Cristo ,videat [...] universam conditionem ex visibilibus intelligens invisibilia et ex creaturis sentiens creatorem"; e dopo aver ricordato Gen. 12, 1, proseguì: „Nemo dubitat patrem nostrum, antequam adoptaremur a deo, fuisse diabolum"; solo dopo essersi spogliata di questo legame antico con il diavolo, assumendo il giogo dell'umiltà, Principia aveva potuto offrirsi all'amore di Dio, suo Signore ${ }^{98}$

- Confusionis urbem. L'etimologia di Babilonia era nota a Girolamo fin dal 375376 quando ne scriveva ai compagni Cromazio, Giovino ed Eusebio ${ }^{99}$. L'origine del termine è spiegata diffusamente nel De situ et nominibus locorum Hebraicorum, composto nel 390: „Babel, quae et Babylon, interpretatur confusio. Erat autem civitas regni Nemrod, in qua eorum qui aedificaverunt turrim, linguae divisae sunt, quorum principem Josephus Nemrod fuisse affirmat. Testem quoque eius historiae Sybillam graecam exhibens: Locus inquit, in quo turrim aedificavere, nunc appellatur Babylon, propter confusionem sermonis eorum, qui altissimam turrim omni studio fabricabantur. Siquidem Hebraei confusionem Babel vocant. De hac turre et diversitate linguarum scribit et Sybilla in hunc modum: Quum omnes mortales una lingua uterentur, quidam ex his altissimam turrim aedificavere, caelum per eam cupientes scandere: dii vero turbines ventosque mittentes, evertere turrim et propriam atque diversam unicuique tribuere linguam: unde urbem quoque apellari contigit Babylonem"100.

${ }_{95}$ Cfr. Dydimus, In Genesim XII 1-3, SCh 244, 136-143.

${ }^{96}$ Cfr. F. Cavallera, Saint Jérôme. Sa vie et son oeuvre, I, Louvain - Paris 1922, 59-62, ibidem, II, pp. 20-22; J.N.D. Kelly, Jerome. His life, writings and controversies, pp. 68-79.

97 C. Moreschini, Praeceptor meus. Tracce dell'insegnamento di Gregorio Nazianzeno in Girolamo, in: Jérôme entre l'Orient et l'Occident, Paris 1988, 137-138.

${ }^{98}$ Cfr. Hieronymus, Epistula 65, 16, CSEL 54, 639.

99 Cfr. F. Cavallera, Saint Jérôme. Sa vie et son oeuvre, II, Louvain - Paris 1922, 154.

${ }^{100}$ De situ et nominibus Hebraicorum. De Genesi, PL 23, 877-878; per la data di composizione cfr. P. Nautin, L'activité litteraire de Jérôme de 387 à 392, pp. 247-259. 
- Roboth. La spiegazione del termine si trova nel De situ et nominibus locorum Hebraicorum: , Robooth civitas Assyriorum, quam aedificavit Assur, egrediens de terra Sennaar" ${ }^{101}$; e nel Liber interpretationis Hebraicorum nominum: "Roboth, inclinatio vel plateae" ${ }^{102}$.

- Sennaar. Cfr. De situ et nominibus locorum Hebraicorum: „Sennaar, campus Babylonis, in quo turris extructa est. Unde egressus Assur aedificavit Ninivem. Meminit huius loci Josephus in primo Antiquitatum libro, ita scribens: De campo qui in Babylonia regione vocatur Sennaar, narrat Hestiaeus historicus, dicens: Quidam sacerdotum profugi de templo Enyalii Iovis sacra rapientes, in Sennaar campum Babyloniac pervenere" ${ }^{103}$.

- Saeculi. Contrapposto a spiritus, è sinonimo di mundus ${ }^{104}$. L'immagine ricorre altrove negli scritti di Girolamo: „Inter minaces saeculi fluctus”105; „Quicumque a deo recedit statim saeculi fluctibus quatitur" ${ }^{106}$; i figli di Abramo „pertranseunt [...] mundi istius turbines”107; „Rubrum huius saeculi pelagus transfretare" 108 .

- Sion. Dal Salmo 136, 1, Girolamo trasse il versetto 4 per esclamare nel momento dell'addio a Roma: „Stultus ego, qui volebam cantare canticum domini in terra aliena et deserto monte Sion Aegypti auxilium flagitabam"109. Sul piano dell'esegesi spirituale per Girolamo Sion e Gerusalemme equivalgono all'idea della chiesa ${ }^{110}$. Il significato etimologico è riportato altrove come „Sion specula vel speculator sive scopulus”" ${ }^{111}$.

- Hiezechiel. Nel commento a Ezechiele, dedicato ad Eustochio e risalente agli anni 410-414, Girolamo riferisce il significato duplice del termine ebraico: „Pro cincinno Septuaginta $œ \propto \alpha \sigma \pi \eta \delta o v$, id est fimbriam, interpretati sunt, quorum alterum in capillis, alterum in vestibus accipi solet"112.

\footnotetext{
${ }^{101}$ De situ et nominibus locorum Hebraicorum. De Pentateucho, PL 23, 915 C

102 Liber interpretationis Hebraicorum nominum. De Genesi, CCL 72, 70.

103 De situ et nominibus locorım Hebraicorım. De Genesi, PL 23, 918 B.

104 Cfr. commento alla voce infra.

105 Hieronymus, Epistula 7, 3, CSEL 54, 28.

106 Idem, Epistula 21, 8, CSEL 54, 119.

107 Idem, Epistula 22, 19, CSEL 54, 168.

108 Idem, Ad Galatas, praefatio, PL 26, 307A.

109 Idem, Epistula 45, 6, CSEL 54, 327.
}

110 Cfr. idem, In Zachariam I 1, 14/16-17, CSEL 76/A, 758-760: „Potest Hierusalem et Sion, visio pacis et specula, quae non bella huius saeculi, nec humilia atque terrena, sed pacem atque concordiam, et caelorum excelsa considerat, Ecclesia intellegi [...]. Quidam consolatam Sion, et electam Hierusalem et cetera quae in hunc modum a prophetis omnibus praedicantur, ad caelestem Hierusalem referunt, quae est destructa per ruinam, per virtutes aedificanda est"; cfr. A. Penna, Principi e carattere dell'esegesi di san Gerolamo, pp. 101, 114-115.

111 Hieronymus, Liber interpretationis Hebraicortum nominum. II Regum, CCL 72, 108, ma anche pp. 112, 122, 153, 157, 161; idem, Epistula 108, 9, CSEL 55, 314-315.

112 Idem, In Hiezechielem III 8, 3, CCL 75, 93. 
- Terram repromissionis. Molti dei temi e dei riferimenti scritturistici dell'Epistula 46 sono ripresi nella lettera a Dardano interamente dedicata alla terra promessa. In essa Girolamo ribadisce che i santi di questa terra, dai Giudei ritenuta la terra promessa, non sono abitanti in senso proprio, ma stranieri che vi abitano temporaneamente. La Palestina è divenuta la terra promessa anche per $\mathrm{i}$ cristiani in virtù della passione e risurrezione di Gesù Cristo ${ }^{113}$.

- Deorsum... sursum. La terra promessa vista da Ezechiele non è l'Egitto, simbolo del mondo terreno, ma la Gerusalemme celeste: „Elevatur a spiritu, qui spiritus non eum statim ad caelum levat, sed inter terram et caelum, ut terrena interim dereliquens festinet ad caelum. Possumus autem et hoc dicere, quod propter bona opera, propheta similitudine manus Dei apprehensus sit, et propter scientiam rerum spiritalium elevatur a spiritu"114.

- Temporaneum... imbrem. Nel commento a Geremia, l'ultimo in ordine di tempo (414-416), dedicato ad Eusebio di Cremona, Girolamo ripete la stessa espressione; poi, riprendendo le antiche traduzioni di Aquila e di Simmaco, chiosa il versetto 5,24 del profeta sottolineando l'ambiguità del termine ebraico sabaoth che talora significa settimana, talaltra pienezza: „Metuamus eum, qui dat nobis pluviam temporaneam et serotinam! per quae omnia ostendi bona plenitudinem annuae messis, pro quo Aquila prima aeditio et Symmacus ebdomades interpretati sunt; in Hebraco enim scriptum est sabaoth, quod pro ambiguitate verbi et septimanas significat et plenitudinem" 115 .

- Terra montuosa et in sublimi sita. Gerusalemme siede sull'altipiano centrale della Palestina ad un'altezza di circa 770 metri sul livello del mare Mediterraneo e di 1165 metri su quello del Mar Morto ${ }^{116}$.

- Maria. Nel 383 a Roma circolò un libro in cui si sosteneva che Maria era stata vergine solo prima della nascita di Gesù. L'autore era Elvidio, discepolo di Aussenzio, vescovo ariano di Milano. Egli ammetteva che i cosiddetti „fratelli di Gesù", di cui parla il Vangelo di Matteo $(12,46 ; 13,55$ ss.) erano stati il frutto di normali rapporti coniugali di Maria con Giuseppe. Il libello si prefiggeva di polemizzare con il modello ascetico della verginità di cui Girolamo era il principale esponente a Roma. Con una disamina acuta dei vangeli canonici, Girolamo difese la verginità di Maria sferrando un feroce attacco contro Elvidio e confutandone l'eresia ${ }^{117}$. L'appellativo $\Theta \varepsilon o$ tóxos, già in uso ai tempi di

${ }^{113}$ Cfr. idem, Epistula 129, 3-4, CSEL 56, 167-171.

114 Idem, In Hiezechielem III 8, 3, CCL 75, 93.

115 Idem, In Hieremiam II 3, 4, CCL 74, 60.

116 Cfr. D. Baldi, Guida di Terra Santa, Gerusalemme 1973, 43.

117 Cfr. Hieronymus, Adversus Helvidium, PL 23, 183-206, per la datazione, la trasmissione del testo e il commento dottrinale cfr. G. Rocca, L'Adversus Helvidium di san Girolamo nel contesto della letteratura ascetico-mariana del secolo $I V$, Bern 1998, da integrare con I . Opelt, Hieronymus' Streitschriften, Heidelberg 1973, 28-36; cfr. Hieronymus, Epistula 77, 2, CSEL 55, 38 : "Virgo puerpera Deum fudit infantem"; idem, Homilia in Iohannem I 1, 14, CCL 78, 521: „Sancta 
Origene, acquisì valore dogmatico al concilio di Efeso nel 431. L'immagine dell'utero come dimora del Figlio di Dio appartiene al linguaggio realistico e allude al passo del vangelo di Giovanni $(2,21)$ in cui si dice che il corpo di Cristo è come il tempio in cui ha preso dimora la divinità del Logos, un concetto ripreso poi da Apollinare di Laodicea ${ }^{118}$.

- Perrexit. Origene aveva insistito sulla necessità per Maria di salire sulla montagna per restare in alto; il termine usato da Girolamo, nella sua traduzione di ỏv $\alpha \beta a i v \varepsilon ı v$ dalla perduta omelia origeniana, è conscendere che sviluppa un concetto caro alla tradizione platonica, ed ora fatto proprio da quella monastica: salire sulla montagna indica il progresso spirituale; restare sulle alture indica lo stato di perfezione, la contemplazione del Logos ${ }^{119}$. Già a Roma, Blesilla, conscia dell'importanza dell'insegnamento ascetico di quei testi, aveva sollecitato a Girolamo la traduzione dei 25 libri di Origene su Matteo, dei 32 su Giovanni e dei 5 su Luca $^{120}$. Ma solo nel 392 Paola ed Eustochio, grazie alle loro insistenze, avevano costretto Girolamo alla pubblicazione in latino di almeno 39 omelie su Luca. A quel tempo la madre e la figlia avevano appreso a "salire sulla montagna" e a contemplare il Logos nella sua divina bellezza. Origene infatti aveva detto che „per coloro che hanno ricevuto il potere di seguirlo (sc. Cristo) anche quando sale sull'alta montagna, c'è una forma più divina"121. Girolamo aveva appreso questo insegnamento allegorico la prima volta a Costantinopoli, dove Gregorio di Nazianzo aveva pronunciato un discorso tra il luglio e il novembre del 380 sviluppando il tema della salita sulla montagna come metafora dell'approccio alla teologia e alla conoscenza del dogma trinitario ${ }^{122}$. In seguito, approfondendo la dottrina origeniana, egli sviluppò la stessa metafora come ascesi monastica ${ }^{123}$.

Maria, beata Maria, mater et virgo, virgo ante partum, virgo post partum"; cfr. A. Penna, San Gerolamo, Roma 1949, 93-99.

118 Testo e trad. it. a cura di M. Simonetti in: Il Cristo, II, Milano 1986, 318-319.

119 Altro riferimento in Io. 13, 3. Cfr. Origenes, Homilia in Lucam VII 2, SCh 87, 154-156, idem, Contra Celsum II 64, SCh 132, 433 passim; ibidem IV 16, SCh 136, 220; ibidem VI 68, 77, SCh $147,348,350,370,372,374$; l'idea era già in Platone, Respublica VII 519 D, e venne ripresa in modo originale nel Corpus Hermeticum X 25, dove si afferma che „l'uomo intero, anima e corpo, pud

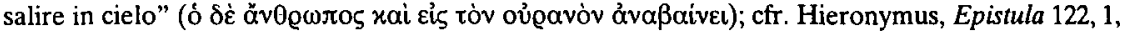
CSEL 55, 56-57, dove è citato l'esempio di Lot.

120 Sul numero esatto dei libri seguo la correzione proposta da P. Nautin, Origène, sa vie et son oeuvre, Paris 1977, 242-243), il quale ipotizza un errore nella trasmissione del testo.

${ }^{121}$ Origenes, Contra Celsum VI 77, SCh 147, 372.

122 Cfr. Gregorius Naz., Oratio 28, 2-3, SCh 250, 14, n. 2 (introduzione di P. Gallay), 102-107 (testo). Girolamo partecipò al concilio di Costantinopoli svoltosi fra il mese di maggio e l'inizio di luglio del 381, cfr. H. Lietzmann, PLRE, VIII 2, 1566-1567, s.v. Hieronymos; F. Cavallera, Saint Jérôme. Sa vie et son oeuvre, I, Louvain - Paris 1922, 59-62; H. Grützmacher, Hieronymus. Eine biographische Studie zur alten Kirchengeschichte, I, Darmstadt 1969, 177-181; J.N.D. Kelly, Jerome. His life, writings and controversies, pp. 68-72.

123 Cfr. L. Mirri, La dolcezza nella lotta. Donne e ascesi, Magnano (Biella) 1996, 174. La metafora del monte (mons est tentatio tua) fu utilizzata da Tommaso da Celano per spiegare il 
- Allophylo. Il neologismo ( $\alpha \lambda \lambda \partial \dot{\phi} \phi \cup \lambda \circ \varsigma$ ) ampiamente attestato in Tertulliano e negli scrittori cristiani dei secoli IV-V si riferisce al filisteo Golia. Nei Settanta il termine greco $\Phi ا \lambda_{L} \sigma \tau \varepsilon i \mu$ riccorre solo in Pentateuco $(46,18)$, Giosué $(48,7)$, Ecclesiaste $(50,28)$ e Maccabei $(I, 4,24)$, negli altri libri dell'Antico Testamento è impiegato invece il termine 'A $\lambda \lambda$ ó $u \lambda$ ol (Alienigenae), mentre nel Nuovo Testamento i Filistei non sono mai menzionati ${ }^{124}$.

- Diabolicae... audacia. Il nemico filisteo è sfrontato come il diavolo. La simbologia che identifica David con Cristo e Golia con il demonio ha origine antiche e trova $\mathrm{i}$ suoi fondamenti nei trattati di Ippolito ${ }^{125}$, Ilario di Poitiers $^{126}$ e Agostino ${ }^{127}$. Con un efficace realismo è rievocata la caduta del gigante Golia e la sua uccisione da parte del giovane David salutato vittorioso con canti e balli dalle donne di Israele (1Reg. 18, 6-7).

- Orna. La costruzione dell'altare nell'area appartenuta ad Ornan rievoca l'antico sacrificio di espiazione per la peste inviata dal Signore che non aveva gradito il censimento degli Israeliti voluto da David. Solo dopo che David ebbe pagato il terreno ad Ornan, cessò il flagello divino (1Par. 21; 2Sm. 24). In seguito, in quel luogo del monte Moria "Salomone cominciò ad edificare la casa del Signore" (2Par. 3, 1).

- Consurgetem. Girolamo interpreta la devastazione di Israele come un segno antigiudaico e accoglie quell'idea consolidata presso i Gentili secondo cui la chiesa sarebbe sorta fra loro, poiché Dio avrebbe punito il popolo di Israele. Rispetto a questa interpretazione, Gerusalemme è vista come „un tipo costante della chiesa" e Israele come la terra santa lasciata in eredità ai Gentili. Gli altri semiti, Idumei, Filistei, Moabiti, Ammaniti, iuxta litteram sono i popoli limitrofi, iuxta tropologia sono gli eretici ,qui tangunt haereditatem Dei et devastant eam" 128 .

miracolo delle stigmate di Francesco sul monte della Verna, cfr. II Tom. Cel., pars II, cap. 81, par. 115 [„,Analecta Franciscana”, X, pp. 198-199] e il commento di C. Frugoni, Francesco el'invenzione delle stigmate", Torino 1993, 137-201.

${ }_{124}$ Cfr. Hieronymus, Epistula 18 A, 1, CSEL 54, 75, dove si cita 1Reg. 17, 49; idem, Epistula 69, 6, CSEL 54, 690, dove si cita Gen. 26, 14-22; cfr. G. Claesson, Index Tertullianaeus, Paris 1974, 91, s.v. allophylus; cfr. TLL, I, 1692, s.v. allophylus; E. Forcellini, Lexicon Totius Latinitatis, I, p. 192, s.v. allophylus.

${ }_{125}$ Cfr. Hippolytus, De David et Goliath VII, CSCO 264, 7.

126 Cfr. Hilarius, Tractatus in Ps. CXLIII 1, CSEL 22, 814: „Intellegimus septuaginta hos seniores sensum nobis spiritalis scientiae reliquisse, ut in David et Golia bellum praefiguratum esse satanae dominique sciremus".

${ }_{127}$ Cfr. Augustinus, Commento ai salmi, a cura di M. Simonetti, Milano 1988, 532-581, 695705 .

${ }^{128}$ Hieronymus, In Sophoniam III 1-7, CCL 76/A, 696; idem, In Hieremiam III 11, CCL 74 , 125; cfr. A. Penna, Principi e carattere dell'esegesi di san Gerolamo, p. 101, n. 1. Se si accettasse la variante urbem, espunta a mio giudizio correttamente da Hilberg, cadrebbe l'interpretazione antigiudaica e bisognerebbe intendere il passo come un riferimento storico alle devastazioni di Tito e di Adriano! 


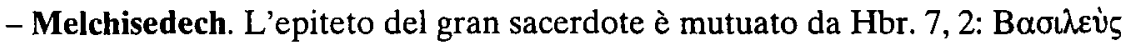

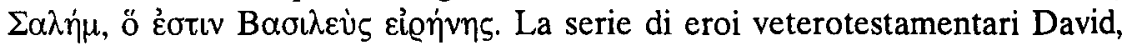
Ornan e Melchisedek, citati a gloria di Gerusalemme, segna una climax figurale: David prefigura la vittoria, Ornan il tempio, Melchisedek il Cristo. Il tema eucaristico e cristologico è di matrice giudeo-cristiana e deriva dal trattato Sul Cristo e sulle chiese, tradizionalmente attribuito a Barsabeo di Gerusalemme, ma ascrivibile forse a Melitone di Sardi: „Riceviamo ancora la testimonianza sul sacrificio e la bevanda del vino e sapremo quel che ne è di Melchisedek che è il Cristo che attende Abramo. Andava in effetti a benedire Abramo quando presentò il pane e il vino"129. Il codice sinaitico n. 508 risalente al secolo IX, noto come Il Libro delle rivelazioni o Caverna dei Tesori ${ }^{130}$, racconta che Melchisedek fu scelto dal Signore come sacerdote sul Golgota, dove era sepolto il corpo di Adamo; gli fu vietato di sposarsi e gli fu detto di sacrificare al Signore solo „pane bianco puro e bevanda del succo di vite". Il testo arabo precisa che Melchisedek era un uomo, non un dio, nato da un padre e da una madre. Diversamente, nella comunità di Qumrân è attestata la tradizione della sua natura celeste; sia Origene che Didimo il Cieco lo dichiarano un angelo ${ }^{131}$; Anche le fonti gnostiche ribadiscono la sua funzione soteriologica ${ }^{132}$; Epifanio di Salamina, la cui influenza su Girolamo appare determinante su questo punto, non risparmiò critiche alla setta che adorava Melchisedek come una divinità e lo riteneva addirittura maggiore di Cristo; costoro, egli dice "sognano" ( $\phi \alpha v \tau \alpha \dot{\zeta}$ ovol) senza aver capito niente, poiché insistono a interpretare letteral-

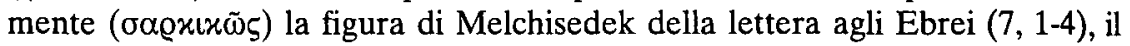
quale fu sì un uomo giusto e un sacerdote di Dio, ma fu un mortale, non il figlio di Dio"133. In quanto legato al Golgota tuttavia, il culto di questo personaggio biblico fu molto forte presso la comunità dei giudeo-cristiani; i quali credevano fermamente al legame fra l'antico sacerdote e il nuovo Messia: ,nel posto stesso in cui Melchisedek esercitava il sacerdozio, là dove Abramo offrì l'agnello sull'altare, è là che fu fissato il legno della croce"134; nello stesso punto localizzavano il suo altare ancora i pellegrini medioevali ${ }^{135}$. Girolamo tuttavia, come

${ }^{129}$ Bersabée de Jérusalem, Sur le Christ et les églises, éd. du texte géorgien inédit et trad. fran. par M. van Esbroeck, PO 41, fasc. 2, n. 187, 175 (introd.), 225 (trad.).

130 Sotto questo titolo sono raccolti diversi scritti apocrifi, antichi commentari biblici, parti di testi leggendari ed apologetici che hanno come temi principali la tomba di Adamo, cioè la Caverna, e il paradiso terrestre, cioè i Tesori: cfr. B. Battista - A. Bagatti (a cura di), La caverna dei tesori, Gerusalemme 1979, 15, 64; La caverne des trésors. Les deux recensions syriaques, traduites par SuMin Ri, CSCO 487, 12-27.

131 Cfr. Hieronymus, Epistula 73, 2, CSEL 55, 14.

132 Cfr. C. Gianotto, DPAC II 2204-2205 (Melchisedek).

133 Epiphanius, Adversus haereses II 56, PG 41, 971. 990.

134 La caverne des trésors. Les deux recensions syriaques, traduites par Su-Min Ri, CSCO 487, 156 (trad. it. dello scrivente dalla versione francese del testo siriaco).

135 Cfr. Antoninus Placentinus, Itinerarium 19, CCL 175, 138-139: „A monumento [sc. Martyrio] 
Epifanio, rigettò l'eresia, pur riconoscendo a Melchisedek il titolo di rex iustitiae e il suo valore di figura cristologica come ,beato apostolo degli Ebrei e dei Gentili" ${ }^{136}$. In seguito egli tentò perfino di dimostrare che la Salem di cui Melchisedek era stato re non corrispondeva a Gerusalemme ma alla vicina Scytopoli, dove si vedevano ancora le rovine del suo grandioso palazzo ${ }^{137}$.

- Typo. Il termine typus, usato anche prima degli autori cristiani, è un calco dal greco e designa all'origine il colpo del martello sul metallo ${ }^{138}$, l'impronta del castone dell'anello $^{139}$; solo in seguito esso passa ad indicare l'idea di stampo e di figura in rilievo, modellata o scolpita, più precisamente di bassorilievo ${ }^{140}$. Dal punto di vista grammaticale typus assume il significato di archetipo, modello, carattere riconoscibile, tipo, così come in Platone (Respublica 396 A). Tertulliano, che usa spesso figura come suo sinonimo, è il primo autore cristiano ad impiegare in latino il termine ${ }^{141}$ nel De idolatria $(24,2)$ e nel De exhortatione castitatis $(6,1)$. Nel secolo IV il neologismo diventa di uso corrente nella terminologia dottrinale dei padri della chiesa. L'esegesi tipologica ,constiste [...] nel riconoscere in alcune realtà dell' Antica Alleanza delle prefigurazioni di realtà della Nuova"; la dottrina tipologica di Girolamo è più vicina alla scuola antiochena che a quella alessandrina, pur esercitando un ruolo di mediazione fra le due scuole; nella prima infatti il typus è distinto dall'allegoria e dal simbolo, perché „deve essere costituito da un vero fatto storicamente accertabile" in rapporto di somiglianza ,perspicua, ma non totale" con il modello che rappresenta ${ }^{142}$. Girolamo si esprime così nel suo commentario ad Osea: „Typus enim partem indicat; quod si totum praecedat in typo, iam non est typus, sed historiae veritas appellanda est" ${ }^{143}$; egli individuò nel tipo un carattere specificamente provvidenziale: „Et hunc esse morem Scripturae sanctae ut futurorum veritatem praemittat in typis" ${ }^{\prime 14}$.

usque Golgotha sunt gressi LXXX. Ab una parte ascenditur per gradus, unde Dominus noster ascendit ad crucifigendum. Nam et locus, ubi crucifixus fuit, paret et cruor sanguinis paret in ipsa petra. In latere est altarium Abrahae, ubi ibat Isaac offerre, obtulit et Melchisedech sacrificium. Ad ipsum altarium est creptura, ubi ponis aurem et audis flumina aquarum et iactas melum aut quod potest natare et vadis in Siloa fonte et ibi eum suscipis. Inter Siloam et Golgotha credo esse miliarum."

136 Hieronymus, Liber interpretationis Hebraicorum nominum. De Genesi, CCL 72, 69; idem, Hebraicae quaestiones in Genesin 14, 18, CCL 72, 19.

${ }^{137}$ Cfr. idem, Epistula 73, 7, CSEL 55, 20: „et ostentatur ibi palatium Melchisedech ex magnitudine ruinarum veteris operis ostendens magnificentiam".

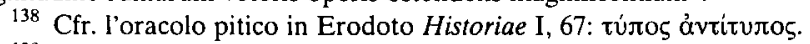

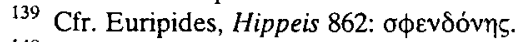

${ }^{140}$ Cfr. Erodoto, Historiae II 136; Plinio, Naturalis historia XXXV 151.

141 Cfr. Tertullianus, De idolatria 24, 4, CCL 2, 1124; idem, De exhortatione castitatis 6, 1, CCL.

$2,1023$.

${ }^{142}$ Cfr. P. Jay, L'exégèse de saint Jérôme, pp. 282, 260-265, su typus in generale.

143 Hieronymus, In Oseam III 11, 1, CCL 76, 122.

144 Idem, In Danielem IV, 11, 21, CCL 75 A, 915. Sull'eclettismo di Girolamo esprime un giudizio positivo A. Penna, Principi e carattere dell'esegesi di san Gerolamo, pp. 1-4, 125-146. 
- Mysterium. Nella definizione stessa di Girolamo „quicquid occultum est et ab hominibus ignoratur, posse mysterium nuncupari" ${ }^{145}$. Fin da Cipriano è attestato l'uso di mysterium nel senso di „figura profetica" „simbolo", ma con una connotazione di oscurità che l'altro suo sinonimo, typus, non possiede. La peculiarità del vocabolo si chiarisce ancor meglio accostandolo a sacramentum, con cui può esserc sostituito: mentre il primo ,mette l'accento sul mistero”, il secondo invece insiste ,sul suo aspetto sacro piuttosto che sul suo aspetto nascosto"146. L'impiego del verbo dedicare specifica il valore sacrale dell'atto inaugurale di Melchisedek; egli fu il primo a rilevare il mistero eucaristico e cristologico nascosto nel sacrificio per mezzo del pane e del vino. Girolamo sottolinea altrove il carattere di purezza del sacrificio eucaristico di Melchisedek, concordando con quanto riporta il testo arabo della Caverna dei Tesori ${ }^{147}$. Egli diede un'interpretazione anagogica del corpo e del sangue di Cristo nel commentario all'Ecclesiaste: „Porro, quia caro Domini verus est cibus et sanguis eius verus est potus, iuxta $\alpha$ v $\alpha \omega \gamma \eta \dot{v} v$ hoc solum habemur in praesenti saeculo bonum, si vescamur carne eius cruoreque potamur, non solum in mysterio, sed etiam in scripturarum lectione"148. Il corpo e il sangue di Cristo costituiscono l'argomento della quarta catechesi mistagogica attribuita a Cirillo di Gerusalemme, che Paola ed Eustochio fecero in tempo a conoscere, dato che la morte del vescovo è fissata fra il 386 e il 387 . Cirillo, riprendendo le parole di Paolo ai Corinzi $(\mathrm{I} 11,23)$, aveva predicato che ,sotto la figura del pane ti è donato il corpo e sotto la figura del vino ti è donato il sangue, affinché tu divenga, avendo partecipato del corpo e del sangue di Cristo, un solo corpo e un solo sangue con il Cristo. Così diventiamo dei portatori di Cristo [...] noi veniamo associati alla natura divina"; e proseguiva parlando di „pane celeste"

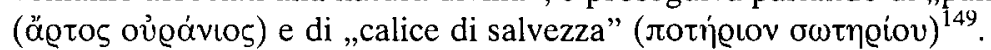

$$
\text { continuazione segue }
$$

${ }^{145}$ Hieronymus, In Danielem II 27, CCL 75 A, 790; cfr. G.J.M. Bartelink, Hieronymos, Liber de optimo genere interpretandi (Epistula 57). Ein Kommentar, Lugduni Batavorum 1980, 45-46; D. Brown, Vir trilinguis. A Study in the biblical exegesis of Saint Jerome, p. 16: „The allegorical interpretation of biblical texts spread into Christianity through Philo, whose great influence led to the development of the catechetical school at Alexandria [...] For Clement, nearly all scripture was expressed in enigmas, and it was the task of the interpreter who had received the deeper knowledge ( $\gamma v \tilde{\omega} \sigma \iota \varsigma)$ given by Christ to his apostles after his resurrection, to unlock the spiritual truth of biblical language to those capable of understanding".

${ }_{146} \mathrm{P}$. Jay, L'exégèse de saint Jérôme, pp. 270, 266-269.

147 Cfr. Hieronymus, Ad Galatas III, 5, PL 26, 417 D: „In Christi sanguinem vinum consecrari”; idem, Epistula 73, 3, CSEL 55, 16: „Pane et vino, simplici puroque sacrificio, Christi dedicaverit sacramentum".

148 Idem, In Ecclesiasten III 12-13, CCL 72, 278. Sui limiti ermeneutici dell'interpretazione anagogica in Girolamo, che scambia frequentemente il termine ỏva $\gamma \omega \gamma \eta \dot{\eta}$ con intellegentia spiritalis. cfr. A. Penna, Principi e carattere dell'esegesi di san Gerolamo, pp. 117-123

${ }_{149}$ Cfr. Cyrillus Hierosolymitanus, Catechesis XXII (Mystagogica IV) 2-6, PG 33, 1097-1101, SCh 126, 136-139. Sul sacrificio eucaristico cfr. 1Cor. 11, 23 passim. 